\title{
Crosstalk between apoptosis and autophagy in prostate epithelial cells under androgen deprivation
}

\author{
RONG-FU LIU* , JIAN LI* , JIE ZHANG, PEI-DE BAI, YU-FENG YANG, WEI LI, ZHUN WU and JIA-XIN ZHENG \\ Department of Urology, The First Affiliated Hospital of Xiamen University, Xiamen, Fujian 361003, P.R. China
}

Received February 3, 2017; Accepted September 19, 2017

DOI: $10.3892 /$ etm.2018.5726

\begin{abstract}
The present study investigated the molecular mechanism of apoptosis and autophagy in prostate epithelial cells under androgen deprivation (AD). BPH-1 cells were divided into four groups as follows: Control (Cont), AD, autophagy inhibition (AI) and AD + AI groups. Cells in the four groups were treated accordingly, and the level of apoptosis was subsequently measured via flow cytometry. The expression of the microtubule-associated proteins 1A/1B light chain 3 (LC3), caspase-3, poly (ADP-ribose) polymerase 1 (PARP-1) and Beclin-1 proteins of BPH-1 cells was detected at different time points following culture in androgen-deprived medium. Western blotting revealed that the basal levels of the LC3-II protein were detected at $0 \mathrm{~h}$. At $4 \mathrm{~h}, \mathrm{LC} 3$-II was significantly increased compared with $0 \mathrm{~h}(\mathrm{P}<0.05)$. Beginning at $20 \mathrm{~h}$, the expression level of the LC3-II protein decreased significantly $(\mathrm{P}<0.05)$. Western blotting revealed that beginning at $24 \mathrm{~h}$, the expression level of the PARP-1 protein decreased significantly $(\mathrm{P}<0.001)$ and the cleavage fragments of the PARP-1 protein appeared. These results further imply that autophagy serves a cell protective function by mutual inhibition with apoptosis in BPH-1 cells in the removal of androgen conditions. Furthermore, the fragments of the cleaved Beclin-1 protein appeared as 35 and $37 \mathrm{kDa}$ bands. Flow cytometry analysis demonstrated that the rate of cell apoptosis in the AD, AI and $\mathrm{AD}+\mathrm{AI}$ groups was significantly increased compared with the Cont group $(\mathrm{P}<0.01)$. Compared with the AD or the AI groups individually, the rate of cell apoptosis in the AD + AI group was significantly increased $(\mathrm{P}<0.001)$. These findings suggest that in the early stage of $\mathrm{AD}$, autophagy has a compensatory function in the cell, whereas in the whole process, autophagy and apoptosis share a mutual antagonism. The Beclin-1-C
\end{abstract}

Correspondence to: Dr Rong-Fu Liu, Department of Urology, The First Affiliated Hospital of Xiamen University, 55 Zhenhai Road, Xiamen, Fujian 361003, P.R. China

E-mail: 1liurf@126.com

${ }^{*}$ Contributed equally

Key words: apoptosis, autophagy, prostate epithelial cells, androgen deprivation, benign prostatic hyperplasia protein fragment contributed positive feedback to the process of apoptosis, which may be a potential mechanism of AD therapy. Therefore, AD and AI exhibit a synergistic effect to further improve the level of apoptosis.

\section{Introduction}

Benign prostatic hyperplasia (BPH) is caused by non-malignant hyperplasia of the prostate. The prevalence of lower urinary tract symptoms due to $\mathrm{BPH}$ increases with increasing age. Moderate to severe symptoms occur in $40-80 \%$ of males $60-80$ years. Nearly all males develop microscopic BPH by the age of 90 years $(1,2) .5 \alpha$-reductase inhibitors (5ARI) are able to reduce the concentration of dihydrotestosterone (DHT) in prostate tissue and thus shrink the prostate (3). Previous studies have revealed that normal prostatic epithelial cells responded to hormone deprivation by undergoing apoptosis (4-6). Over the course of treatment with Finasteride, a $5 \alpha$-reductase inhibitors, serum PSA level is reduced by $50 \%$, and over time total prostate volume decreases by $15-25 \%$ due to apoptosis and shrinkage of the glandular epithelial compartment in the transition and peripheral zones of the prostate (7). However, the molecular mechanism of apoptosis in prostate epithelial cells in the process of androgen deprivation (AD) remains unclear.

Macroautophagy (hereafter, autophagy) is an evolutionarily conserved catabolic process, which is essential in homeostasis and the stress-response as well as in macromolecular turnover and development (8). The association between apoptosis and autophagy is complex. In a number of scenarios, apoptosis and autophagy are able induce cell death in a coordinated or cooperative manner (9). In other cell types and the types of stress, autophagy acts as an antagonist to block apoptotic cell death by promoting cell survival (10). For example, pre-treatment of cells with rapamycin, which induces autophagy and causes a decrease in the mitochondrial mass by $\sim 50 \%$ while reducing the susceptibility of cells to MOMP-dependent apoptotic stimuli (11). In any given death scenario, the fate of the cell is dependent on cellular settings, the nature of the stimulus and the particulars of the cell growth microenvironment (12). Li et al (13) previously reported that autophagy and apoptosis perform in a mutually exclusive manner in $\mathrm{LNCaP}$ prostate adenocarcinoma cells under androgen-deprivation conditions. They also reported that the level of autophagy markedly increased, and that the inhibition of autophagy by 
3-methyladenine significantly promoted the rate of apoptosis in PWR-1E cells under the conditions of AD. Additionally, in vivo, autophagy was demonstrated to significantly increase in hyperplasia of prostate tissue following 5-ARI treatment (14). To the best of our knowledge, no study is currently available on the molecular mechanism of apoptosis and autophagy of prostate epithelial cells undergoing AD.

Beclin-1, which is the mammalian orthologue of yeast Atg6, has a central role in autophagy (12). Wirawan et al (15) previously revealed that Beclin-1 was cleaved by caspase in a cellular model system in which the withdrawal of the growth factor interleukin (IL-3) induced apoptotic cell death that was preceded by a phase of increased autophagy. Additionally, cleavage of Beclin-1 by caspase produced Beclin1-C, which was translocated to the mitochondria and amplified apoptosis induced by IL-3 deprivation. This indicates that the cleavage of Beclin-1 may contribute an amplifying loop for the enhancement of apoptosis (16). The present study proposes that the process of Beclin-1 protein cleavage may serve a role in the apoptosis of benign epithelial cells under AD, and explores whether the cleavage of the Beclin-1 protein leads to the activation of cell apoptosis. The present study also evaluated whether the combination of AD and autophagy inhibition (AI) had an additive or a synergistic effect on the enhancement of apoptosis.

\section{Materials and methods}

Chemicals and antibodies. DHT and chloroquine (CQ) were purchased from Sigma-Aldrich (Merck KGaA, Darmstadt, Germany). Antibodies against microtubule-associated proteins 1A/1B light chain 3 (LC3) (cat no. D3U4C), poly (ADP-ribose) polymerase 1 (PARP-1) (cat no. 46DH), caspase-3 (cat no. 8G10) and GAPDH (cat no. 14C10) were purchased from Cell Signaling Technology, Inc. (Danvers, MA, USA), and antibodies against Beclin-1 were from BD Biosciences (cat no. 612112; Franklin Lakes, NJ, USA). Horseradish peroxidase-labeled goat anti-rabbit secondary antibodies (cat no. GAR007) and anti-rat secondary antibodies (cat no. GAM007) were purchased from MultiSciences Biotech Co., Ltd (Hangzhou, China). The Annexin V-fluorescein isothiocyanate/propidium iodide (FITC/PI) apoptosis kit was purchased from Dojindo Molecular Technologies, Inc. (Kumamoto, Japan).

Cell culture and treatments. BPH-1 cells (Sangon Biotech Co., Ltd., Shanghai, China), an immortalized but non-transformed human prostate epithelial cell line, which was immortalized by infection with the ZipneoSV virus carrying the SV40 T antigen gene (17), were provided by the Department of Urology of the Union Hospital of Fujian Medical University. The cells were maintained at $37^{\circ} \mathrm{C}$ in RPMI 1640 medium (Gibco; Thermo Fisher Scientific, Inc., Waltham, MA, USA) that contained $10 \%$ fetal bovine serum (Gibco; Thermo Fisher Scientific, Inc.). Prior to the corresponding treatment, $\mathrm{BPH}-1$ cells were washed twice with PBS to eliminate the remnant serum. It has previously been demonstrated that the total testosterone of a healthy adult human male was $10-35 \mathrm{nM}(18)$. Li et al $(13,14)$ have previously reported that DHT treatment reduced the level of autophagy, with the highest inhibition observed at $10 \mathrm{nM}$.
BPH-1 cells that were cultured in RPMI 1640 medium with $10 \%$ fetal bovine serum and $10 \mathrm{nM}$ DHT at $37^{\circ} \mathrm{C}$ for $24 \mathrm{~h}$ served as the control group (Cont). Cells cultured in phenol red-free RPMI 1640 medium with 10\% charcoal-stripped fetal bovine serum (Gibco; Thermo Fisher Scientific, Inc.) served as the AD group. Cells cultured in RPMI 1640 medium with $10 \%$ fetal bovine serum, $1 \times 10^{-8} \mathrm{mmol} / 1 \mathrm{DHT}$ and $5 \times 10^{-5} \mathrm{mmol} / 1$ CQ served as the AI group. Finally, cells cultured in phenol red-free RPMI 1640 medium with $10 \%$ charcoal-stripped fetal bovine serum and $5 \times 10^{-5} \mathrm{mmol} / 1 \mathrm{CQ}$ served as the $\mathrm{AD}+\mathrm{AI}$ group. The cells in each group were cultured for $24 \mathrm{~h}$ at $37^{\circ} \mathrm{C}$.

Western blot analysis. Cells were cultured at $37^{\circ} \mathrm{C}$ in phenol red-free RPMI 1640 medium that contained $10 \%$ charcoal-stripped fetal bovine serum, we defined the time for the replacement of the cell culture medium as $0 \mathrm{~h}$, and the total protein was extracted using a radioimmunoprecipitation buffer (25 mM TrisNHCl pH 8, $150 \mathrm{mM} \mathrm{NaCl,} \mathrm{1 \%} \mathrm{NP-40,}$ $0.5 \%$ sodium deoxycholate, $0.1 \%$ SDS) and protease inhibitor cocktail (Sigma-Aldrich; Merck KGaA) every 4 h for a total of $48 \mathrm{~h}$. A BCA protein assay kit was then used as a protein determination method (Thermo Fisher Scientific, Inc). Protein $10 \mu \mathrm{l}(1,500 \mu \mathrm{g} / \mathrm{ml})$ per lane from each sample was then subjected to electrophoresis on an $8 \%$ SDS-PAGE for PARP-1 detection, a 10\% SDS-PAGE for caspase-3 and Beclin-1 detection and a $15 \%$ SDS-PAGE for LC3 detection, and transferred onto polyvinylidene fluoride membranes. Following blocking with $5 \%$ nonfat dry milk for $90 \mathrm{~min}$ at room temperature, membranes were incubated with primary antibodies overnight at $4^{\circ} \mathrm{C}$. The primary antibodies used were as follows: PARP-1 polyclonal rabbit primary antibody $(1: 1,000)$; Caspase-3 primary antibody (1:1,000); LC-3 polyclonal rabbit primary antibody (1:1,000; all Cell Signaling Technology); Beclin-1 polyclonal rat primary antibody (1:2,000; BD Biosciences); and GAPDH polyclonal rabbit primary antibody (1:2,000; both $\mathrm{BD}$ Biosciences) as a reference protein. Membranes were then incubated with corresponding horseradish peroxidase-conjugated secondary antibodies at 1:5,000 dilutions for $2 \mathrm{~h}$ at room temperature. Protein bands were visualized on X-ray film using an enhanced chemiluminescence system (Advansta, CA, USA). LC3-I and LC3-II are 18 and $16 \mathrm{kDa}$ respectively (19). The 35- and 37-kDa Beclin-1 fragments generated during apoptosis are C-terminal Beclin-1 fragments resulting from caspase-mediated cleavage. Densitometry analysis was performed using ImageJ v1.45 software (National Institutes of Health, Bethesda, MD, USA).

Flow cytometry for the measurement of the apoptosis rate of each group. Cells were seeded at $1 \times 10^{6}$ cells in $2.5 \mathrm{ml}$ RPMI 1640 medium containing 10\% FBS per well in 6-well plates for $24 \mathrm{~h}$ at $37^{\circ} \mathrm{C}$ prior to exposure to the corresponding treatment. Following incubation, the cells in each group were exposed to the corresponding treatment for an additional $24 \mathrm{~h}$, and the supernatant medium from each well was transferred in a separate pre-labeled centrifuge tube to collect non-adherent cells. Adherent cells from the same well were then trypsinized and transferred to the same centrifuge tube containing the non-adherent cells. Cells in each centrifuge tube were then centrifuged at $2,000 \mathrm{x} \mathrm{g}$ at $4^{\circ} \mathrm{C}$ for $5 \mathrm{~min}$, and the supernatant was discarded. The cells were re-suspended 
in $100 \mu \mathrm{l}$ Annexin $\mathrm{V}$ binding buffer (Dojindo Molecular Technologies, Inc.). In total, $5 \mu \mathrm{l}$ FITC-Annexin $\mathrm{V}$ and $5 \mu \mathrm{l}$ PI (both from Dojindo Molecular Technologies, Inc.) were added and the cells were incubated at room temperature for $15 \mathrm{~min}$ in the dark. Subsequently, $400 \mu \mathrm{l}$ Annexin V binding buffer was added and flow cytometry was performed on a Beckman flow cytometer (Beckman Coulter, Inc., Brea, CA, USA). Cells were considered to be apoptotic if they were Annexin $\mathrm{V}^{+} / \mathrm{PI}^{-}$(early apoptotic) and Annexin $\mathrm{V}^{+} / \mathrm{PI}^{-}$(late apoptotic). At least 10,000 events were recorded and the data were evaluated using Kaluza 1.3 software (Beckman Coulter, Inc.) for each analysis.

Statistical analysis. All experiments were repeated three times and similar results were obtained. All data of normal distribution are presented as the mean \pm standard deviation and were analyzed by one-way analysis of variance (ANOVA) or two-way ANOVA factorial design. The data of a non-normal distribution were analyzed by the Kruskal-Wallis $\mathrm{H}$ and the Wilcoxon rank-sum tests. When statistical significance was identified, the differences between groups were further analyzed by the Nemenyi test. $\mathrm{P}<0.05$ was considered to indicate a statistically significant difference. SPSS 11.5 (SPSS, Inc., Chicago, IL, USA) was used for all statistical analyses.

\section{Results}

Expression of LC3-II protein with time in BPH-1 cells under $A D$. Autophagy in BPH-1 cells under AD was monitored using the levels of LC3-II compared with GAPDH protein as an autophagy-specific marker and not to that of LC3-I (20). AD was associated with increased autophagy during the first $4 \mathrm{~h}$ as evidenced by significantly increased LC3-II levels (Fig. 1). Consistent with a previous report (21), the basal levels of LC3-II could be detected even in the presence of DHT $(0-\mathrm{h}$ time point in Fig. 1A), which reflects the regulation of cellular homeostasis by autophagy. The expression of the LC3-II protein was significantly decreased at 20 compared with $16 \mathrm{~h}$ (Fig. 1), and this low level was maintained at $24 \mathrm{~h}$. These results indicated that there was a basal level of autophagy activation at $0 \mathrm{~h}$; autophagy was significantly activated at $4-16 \mathrm{~h}$ and the level of autophagy significantly decreased from the beginning of the 20-h time point.

Expression of PARP-1 protein changes with time in $\mathrm{BPH}-1$ cells under $A D$. Protein synthesis and tissue degradation decreased in prostate epithelial cells under AD. Additionally, AD was able to activate cell apoptosis-associated genes, which increase the level of apoptosis of prostate epithelial cells (22). The expression level of the PARP-1 protein in BPH-1 cells that were cultured in an androgen-deprived medium for 0,4 , $8,12,24,36$ and $48 \mathrm{~h}$ was detected by western blot analysis, which was used to detect the level of apoptosis of BPH-1 cells under AD. The hydrolysis fragments of PARP-1 protein were captured at $24 \mathrm{~h}$ and the expression level of PARP-1 protein was almost undetectable at other time points (Fig. 2A). The cause of this phenomenon is not clear, it is speculated that it may be that the transformation of the PARP-1 protein is rapid in this cell type and the type of stress. Thus, the hydrolysis
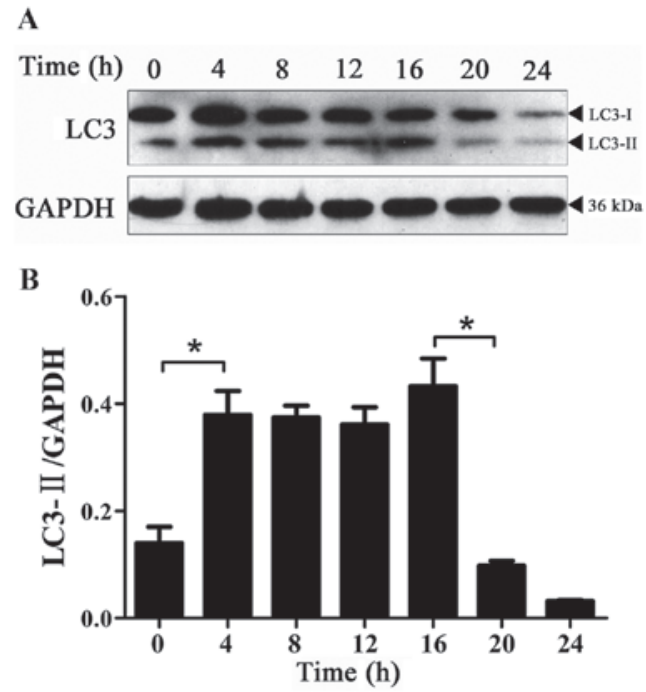

Figure 1. Expression of the LC3-II protein over time in BPH-1 cells under AD. (A) BPH-1 cells were exposed to AD for the indicated durations. Protein samples were prepared and analyzed by western blot analysis using anti-LC3 and anti-GAPDH antibodies. (B) Relative expression of LC3-II proteins in $\mathrm{BPH}-1$ cells under the condition of $\mathrm{AD}$ at each time point was investigated by western blot analysis. Data are representative of the relative expression of proteins that were normalized by GAPDH. Data are presented as the mean \pm standard deviation $(n=3)$. ${ }^{*} \mathrm{P}<0.05$. LC3, microtubule-associated proteins $1 \mathrm{~A} / 1 \mathrm{~B}$ light chain 3 ; $\mathrm{AD}$, androgen deprivation.
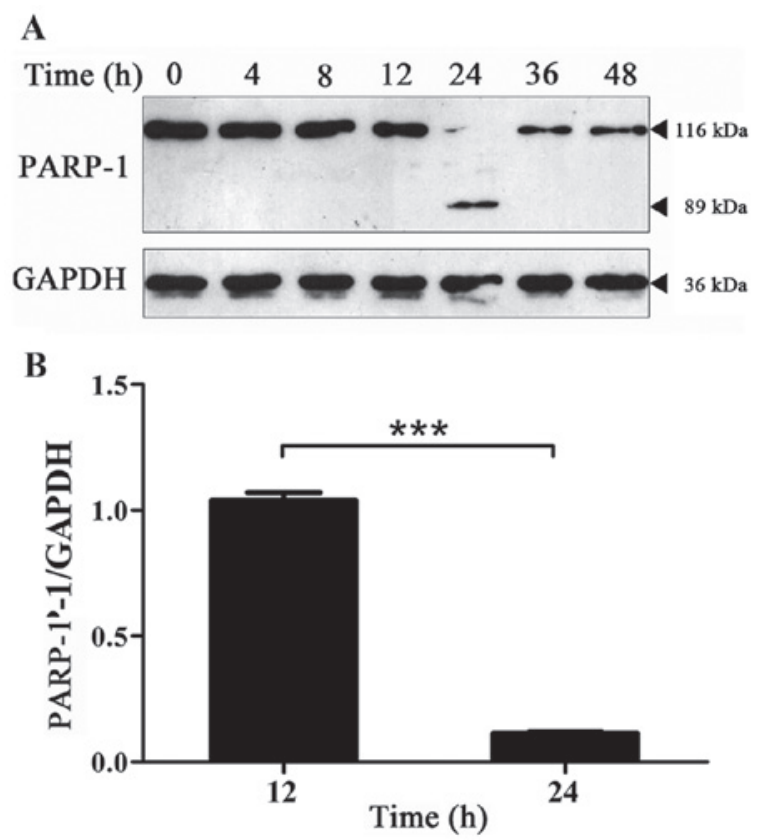

Figure 2. Expression of PARP-1 protein over time in BPH-1 cells under AD. (A) BPH-1 cells were exposed to AD for the indicated durations. Protein samples were prepared and analyzed by western blot analysis using anti-PARP-1 and anti-GAPDH antibodies. (B) Relative expression of PARP-1 proteins in BPH-1 cells under AD at each time point was investigated by western blot analysis. Data are representative of the relative expression of proteins that were normalized by GAPDH. Data are presented as the mean \pm standard deviation $(\mathrm{n}=3)$. ${ }^{* * *} \mathrm{P}<0.001$. PARP-1, poly (ADP-ribose) polymerase 1 ; $\mathrm{AD}$, androgen deprivation.

fragments of PARP-1 protein could be detected only when the hydrolysis of PARP-1 was at a high enough level. Therefore, PARP-1 protein fragments were not used for quantitative 
analysis, but the expression of PARP-1 protein is presented. The results revealed that compared with $12 \mathrm{~h}$, the expression level of the PARP-1 protein was significantly decreased at $24 \mathrm{~h}$ (Fig. 2). These results indicate that there was a significant increase in cell apoptosis at $24 \mathrm{~h}$.

Change in the expression level of LC3-II and PARP-1 in $B P H-1$ cells under $A D$. In the absence of nutrients or cytokines, cell autophagy typically serves a role in cell protection. The protective effect of autophagy on cells is limited, and the main manifestations of apoptosis and autophagy are considered to be mutually antagonistic (10). The present study monitored the changes in apoptosis and autophagy in BPH-1 cells under AD by detecting the expression levels of LC3-II and PARP-1 in the BPH-1 cells that were cultured in an androgen-deprived medium for $0,12,16,20,24,28$ and $32 \mathrm{~h}$. The results revealed that the expression of LC3-II was significantly decreased at $20 \mathrm{~h}$ compared with its expression at $16 \mathrm{~h}$. However, the expression level of PARP-1 was significantly decreased starting at the 20-h time point (Fig. 3). These results indicate that under the conditions of $A D$, the activation of autophagy occurs prior to apoptosis in BPH-1 cells, and the activation of apoptosis occurs when the level of autophagy has decreased.

Caspase-3 protein activation is accompanied by the hydrolysis of the Beclin-1 protein in the process of apoptosis. To determine the expression of the caspase- 3 protein and the hydrolysis of the Beclin-1 protein over time, the expression of the caspase- 3 and Beclin-1 proteins at the corresponding time points was detected by western blot analysis. The results revealed that the expression level of the caspase- 3 protein was markedly decreased at $20 \mathrm{~h}$, and the 35 and 37-kDa hydrolysis fragments of the Beclin-1 protein appeared at the beginning of the 20-h time point (Fig. 4). These results indicate that in the process of BPH-1 cell apoptosis, the Beclin-1 protein is hydrolyzed.

Effect of $A D, A I$ and $A D+A I$ on the level of apoptosis of prostate epithelial cells. The apoptosis rate of each group was monitored using flow cytometry. The results revealed that compared with the Cont group, the apoptosis level of the cells in the $\mathrm{AD}$, the $\mathrm{AI}$ and the $\mathrm{AD}+\mathrm{AI}$ groups were significantly increased (Fig. 5). Furthermore, compared with the AD or AI groups, the apoptosis rate of the BPH-1 cells was significantly increased in the AD + AI group (Fig. 5). The analysis of the two-way ANOVA factorial design indicated that AD and the inhibition of autophagy may have a synergistic effect. These results indicate that $\mathrm{AD}$ or inhibition of autophagy significantly increase the level of apoptosis of BPH-1 cells. Additionally, AD and the inhibition of autophagy may produce synergistic effects and further increase the rate of apoptosis in BPH-1 cells.

\section{Discussion}

When cells suffer extracellular or intracellular stress, including growth factor deprivation, starvation, endoplasmic reticulum stress and pathogen infection, autophagy is always upregulated to serve a role in cytoprotection (23). Androgens
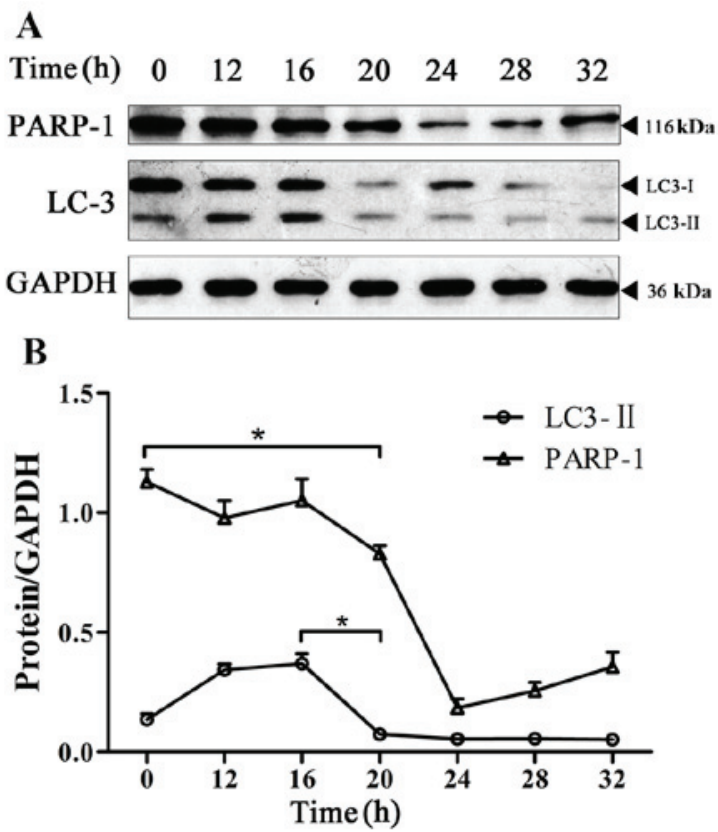

Figure 3. Expression of the LC3-II and PARP-1 proteins over time in BPH-1 cells under AD. (A) BPH-1 cells were exposed to AD for 0, 12, 16, 20 , 24, 28 and $32 \mathrm{~h}$. Protein samples were prepared and analyzed by western blot analysis using anti-LC3, anti-PARP-1 and anti-GAPDH antibodies. (B) Relative expression of the LC3-II and PARP-1 proteins in BPH-1 cells under $\mathrm{AD}$ at each time point was investigated by western blot analysis. Data are representative of the relative expression of proteins that were normalized by GAPDH. Data are presented as the mean \pm standard deviation $(n=3)$. ${ }^{*} \mathrm{P}<0.05$. LC3, microtubule-associated proteins 1A/1B light chain 3; PARP-1, poly (ADP-ribose) polymerase $1 ; \mathrm{AD}$, androgen deprivation.

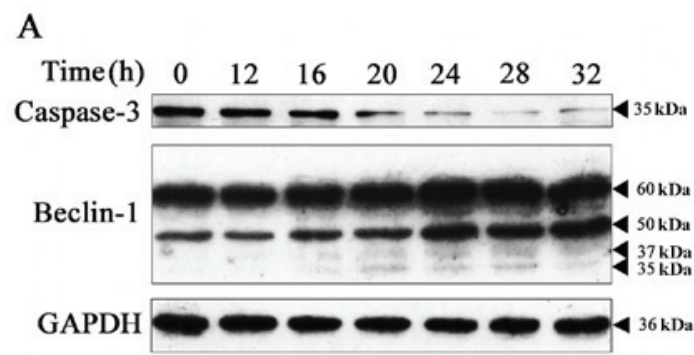

B

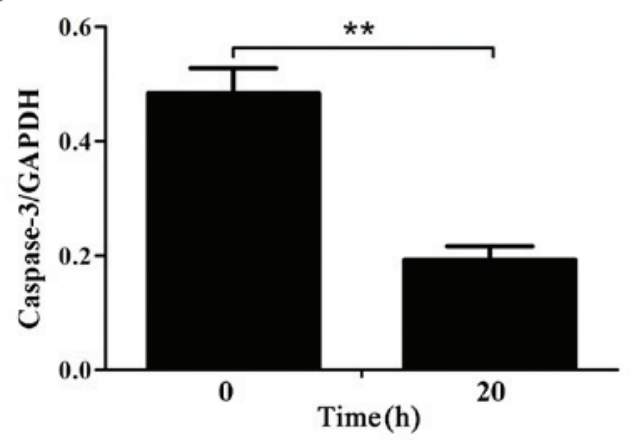

Figure 4. Beclin-1 is cleaved during apoptosis. (A) Whole-cell lysates were prepared from androgen-deprived BPH-1 cells for 0, 12, 16, 20, 24, 28 and $32 \mathrm{~h}$ and analyzed by western blot analysis with anti-Beclin-1, anti-Caspase-3 and anti-GAPDH antibodies. (B) Relative expression of Caspase-3 proteins in BPH-1 cells under AD at 0 and $20 \mathrm{~h}$ was investigated using western blot analysis. Data are representative of the relative expression of proteins that were normalized by GAPDH. Data are presented as the mean \pm standard deviation $(n=3)$. ${ }^{* *} \mathrm{P}<0.01$. Beclin-1, a protein that in humans is encoded by the BECN1 gene; Caspase-3, cysteine-aspartic acid protease-3; AD, androgen deprivation. 
A

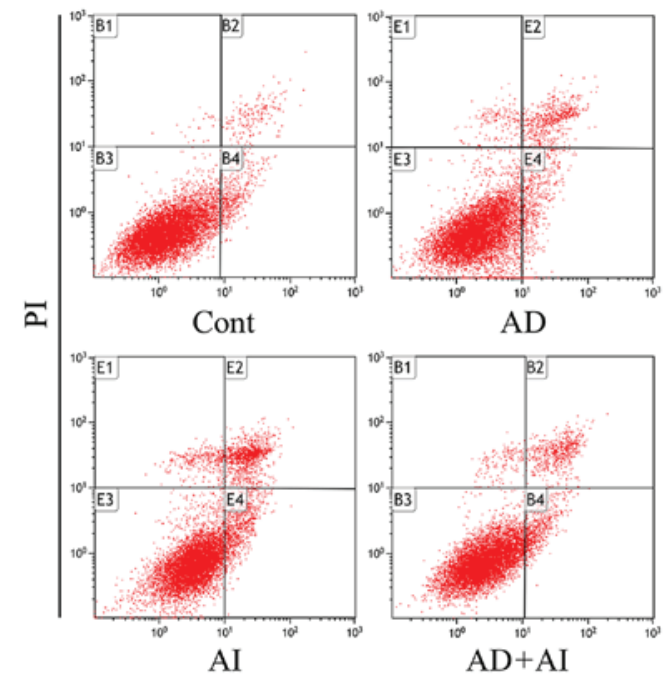

B

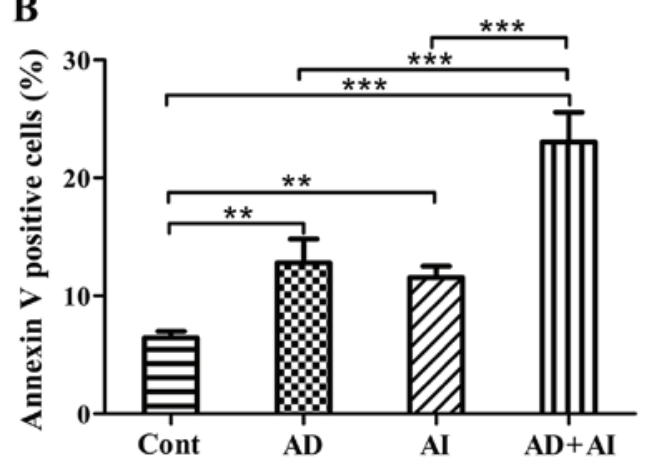

Figure 5. AD and AI exhibit synergistic effects to increase the rate of apoptosis in BPH-1 cells. (A) BPH-1 cells in each group were exposed to corresponding treatments for $24 \mathrm{~h}$. Cell death was measured by flow cytometry labeled with Annexin V/PI-staining. (B) The number of apoptotic/secondary necrotic cells is demonstrated as the sum of Annexin V-positive and Annexin V/PI double-positive cells. Data are presented as the mean \pm standard deviation $(\mathrm{n}=3) .{ }^{* * *} \mathrm{P}<0.01,{ }^{* * *} \mathrm{P}<0.001$. AD, androgen deprivation; AI, autophagy inhibition; PI, propidium iodide; Cont, control.

serve a critical role in prostate development, growth and pathogenesis. The use of 5ARIs and castration are effective therapies for BPH and prostate cancer, respectively (3). To investigate the variation of autophagy in human prostate epithelial cells under AD, a human prostate epithelial cell line, BPH-1, was used, which was cultured in a medium in which the androgens had been removed. The autophagy of BPH-1 cells was monitored at different time points using the processing of cytosolic microtubule-associated protein LC3-I into LC3-II as an autophagy-specific marker (8). The present results demonstrated that autophagy is evidently activated in the early stages of androgen removal, which implies that autophagy is upregulated in response to AD. The results of flow cytometry demonstrated that inhibition of autophagy may further improve the level of apoptosis of BPH-1 cells under AD. These results indicate that autophagy functions as a survival mechanism under AD.

In clinical practice 5-ARI is often used to maximally induce prostate cell apoptosis by blocking the alteration of testosterone to DHT for the treatment of BPH and prostate cancer (3). The association between apoptosis and autophagy is complex in that, depending on the cell type and stimulus, different sensitivity thresholds may dictate whether autophagy or apoptosis may develop; they customarily exhibit some degree of mutual inhibition (12). Initially, the significant activation of apoptosis was confirmed to occur at $24 \mathrm{~h}$ in BPH-1 cells under AD. Subsequently, the combined apoptotic and autophagic results of the present study demonstrated that once apoptosis was initiated in BPH-1 cells, the LC3-II levels were markedly diminished starting at $20 \mathrm{~h}$. These results further demonstrate that autophagy serves a cell-protective function by mutual inhibition with apoptosis in BPH-1 cells in androgen-deficient conditions.

Li et al $(13,14)$ previously reported that the blockage of autophagy by 3 -methyladenine led to increased apoptosis of LNCaP or PWR-1E cells in serum-free medium compared with the medium with DHT. However, the interactive mechanism between cell apoptosis and autophagy remains unclear in this process. Wirawan et al (15) previously reported that the withdrawal of the obligatory growth factor IL-3 from $\mathrm{Ba} / \mathrm{F} 3$ cells, which are dependent on IL-3 for growth, induced apoptotic cell death that was preceded by a phase of increased autophagy. The authors identified that Beclin-1, a component of the autophagy-inducing complex, was a direct substrate of the caspases and that cleavage of Beclin-1 was observed in response to the mitochondrial pathways of apoptosis. Based on these findings, it was hypothesized that the cleavage of Beclin-1 sensitizes cells to apoptotic signals, inhibits cell autophagy and can form an amplifying loop for inducing massive apoptotic cell death (16). The present study investigated whether there was a similar occurrence in BPH-1 cells. As expected, cleavage of Beclin-1 coincided with caspase-3 maturation starting at $20 \mathrm{~h}$. The previous experimental results confirmed that the level of apoptosis was increased and that the autophagy level was decreased starting at $20 \mathrm{~h}$, which indicated a similar molecular mechanism present in BPH-1 cells under AD.

Clinically, 5-ARI has been known to reduce the volume of the prostate; however, the reduced volume is only $\sim 20 \%$, which reduces the long-term risk of the need for surgical therapy by 55\% (24). The treatment options for BPH include medical therapy and surgery. Surgical therapy has certain risks and numerous complications; therefore, it is important to improve the therapeutic effect of drugs to delay the progression of the disease and reduce the risk of surgery (25). Inhibition of autophagy may be a novel therapeutic strategy. According to a report of testosterone levels in the serum from adult human males the normal range is $10-35 \mathrm{nM}$ (18). In the current study, BPH-1 cells were cultured in medium containing $1 \times 10^{-8} \mathrm{mmol} / 1 \mathrm{DHT}$ as the control group. The results of flow cytometry revealed that compared with the normal Cont group, the apoptosis level of the cells in the $\mathrm{AD}$ and $\mathrm{AI}$ groups were significantly increased. The results indicated that AD was able to induce apoptosis, and the inhibition of autophagy could induce apoptosis in prostate epithelial cells. Notably, the rate of apoptosis of prostate epithelial cells induced by AD or AI was similar. Additionally, the experimental data derived from flow cytometry revealed that the cells that are subject to $\mathrm{AD}$ and $\mathrm{AI}$ are in accordance with the two-way ANOVA factorial design. Furthermore, the data analysis results indicated that $\mathrm{AD}$ and the inhibition of autophagy could exhibit a synergistic effect. 
Such results indicate that the combination of AD and AI was able to induce apoptosis of prostate epithelial cells more effectively and are most likely associated with the amplifying loop mechanism derived from the caspase-mediated cleavage fragments of Beclin-1. These results contribute to the further study for the application of autophagy in the treatment of $\mathrm{BPH}$. The present study focuses on the BPH-1 cell line only and therefore, further validation is required in other prostate epithelial and stromal cells, and within studies using animals.

\section{Acknowledgements}

The present study was sponsored by Medical Elite Cultivation Program of Fujian, P.R.C (no. 2014-ZQN-ZD-33).

\section{References}

1. Dhingra $\mathrm{N}$ and Bhagwat D: Benign prostatic hyperplasia: An overview of existing treatment. Indian J Pharmacol 43: 6-12, 2011.

2. Roehrborn CG and McConnell J: Benign prostatic hyperplasia: Etiology, pathophysiology, epidemiology and natural history. In Campbell's Urology. Walsh PC, Retik AB, Vaughan ED Jr and Wein AJ (eds). 8th edition. WB Saunders Co, Philadelphia, PA: pp. 1297-1336, 2002.

3. Moore A, Butcher MJ and Köhler TS: Testosterone replacement therapy on the natural history of prostate disease. Curr Urol Rep 16: 51, 2015.

4. Silva IS, Morsch DM, Urnauer L and Spritzer PM: Androgen-induced cell growth and c-myc expression in human non-transformed epithelial prostatic cells in primary culture. Endocr Res 27: 153-169, 2009.

5. Furuya Y and Isaacs JT: Differential gene regulation during programmed death (apoptosis) versus proliferation of prostatic glandular cells induced by androgen manipulation. Endocrinology 133: 2660-2666, 1993.

6. Raffo AJ, Perlman H, Chen MW, Day ML, Streitman JS and Buttyan R: Overexpression of bcl-2 protects prostate cancer cells from apoptosis in vitro and confers resistance to androgen depletion in vivo. Cancer Res 55: 4438-4445, 1995.

7. Roehrborn CG: Benign prostatic hyperplasia: An overview. Rev Urol 7 (Suppl 9): S3-S14, 2005.

8. Klionsky DJ, Abdelmohsen K, Abe A, Abedin MJ, Abeliovich H, Acevedo Arozena A, Adachi H, Adams CM, Adams PD, Adeli K, et al: Guidelines for the use and interpretation of assays for monitoring autophagy (3rd edition). Autophagy 12: 1-222, 2016.

9. Fimia GM and Piacentini M: Regulation of autophagy in mammals and its interplay with apoptosis. Cell Mol Life Sci 67: 1581-1588, 2010.

10. Maiuri MC, Zalckvar E, Kimchi A and Kroemer G: Self-eating and self-killing: Crosstalk between autophagy and apoptosis. Nat Rev Mol Cell Biol 8: 741-752, 2007.

11. Ravikumar B,BergerZ, Vacher C,O'Kane CJ and Rubinsztein DC: Rapamycin pre-treatment protects against apoptosis. Hum Mol Genet 15: 1209-1216, 2006.
12. Eisenberg-Lerner A, Bialik S, Simon HU and Kimchi A: Life and death partners: Apoptosis, autophagy and the cross-talk between them. Cell Death Differ 16: 966-975, 2009.

13. Li M, Jiang X, Liu D, Na Y, Gao GF and Xi Z: Autophagy protects LNCaP cells under androgen deprivation conditions. Autophagy 4: 54-60, 2008.

14. Li M, Yang X, Wang H, Xu E and Xi Z: Inhibition of androgen induces autophagy in benign prostate epithelial cells. Int J Urol 21: 195-199, 2014.

15. Wirawan E, Vande Walle L, Kersse K, Cornelis S, Claerhout S, Vanoverberghe I, Roelandt R, De Rycke R, Verspurten J, Declercq W, et al: Caspase-mediated cleavage of Beclin-1 inactivates Beclin-1-induced autophagy and enhances apoptosis by promoting the release of proapoptotic factors from mitochondria. Cell Death Dis 1: e18, 2010.

16. Djavaheri-Mergny M, Maiuri MC and Kroemer G: Cross talk between apoptosis and autophagy by caspase-mediated cleavage of Beclin 1. Oncogene 29: 1717-1719, 2010.

17. Hayward SW, Dahiya R, Cunha GR, Bartek J, Deshpande N and Narayan P: Establishment and characterization of an immortalized but non-transformed human prostate epithelial cell line: BPH-1. In Vitro Cell Dev Biol Anim 31: 14-24, 1995.

18. Sedelaar JP and Isaacs JT: Tissue culture media supplemented with $10 \%$ fetal calf serum contains a castrate level of testosterone. Prostate 69: 1724-1729, 2009.

19. Kabeya Y, Mizushima N, Ueno T, Yamamoto A, Kirisako T, Noda T, Kominami E, Ohsumi Y and Yoshimori T: LC3, a mammalian homologue of yeast Apg8p, is localized in autophagosome membranes after processing. EMBO J 19: 5720-5728, 2000.

20. Klionsky DJ, Abdalla FC, Abeliovich H, Abraham RT, Acevedo-Arozena A, Adeli K, Agholme L, Agnello M, Agostinis P, Aguirre-Ghiso JA, et al: Guidelines for the use and interpretation of assays for monitoring autophagy. Autophagy 8: 445-544, 2012.

21. Altman BJ, Wofford JA, Zhao Y, Coloff JL, Ferguson EC, Wieman HL, Day AE, Ilkayeva O and Rathmell JC: Autophagy provides nutrients but can lead to chop-dependent induction of bim to sensitize growth factor-deprived cells to apoptosis. Mol Biol Cell 20: 1180-1191, 2009.

22. Isaacs JT: Antagonistic effect of androgen on prostatic cell death. Prostate 5: 545-557, 1984.

23. He C and Klionsky DJ: Regulation mechanisms and signaling pathways of autophagy. Annu Rev Genet 43: 67-93, 2009.

24. Nickel JC, Gilling P, Tammela TL, Morrill B, Wilson TH and Rittmaster RS: Comparison of dutasteride and finasteride for treating benign prostatic hyperplasia: The Enlarged Prostate International Comparator Study (EPICS). BJU Int 108: 388-394, 2011.

25. McVary KT, Roehrborn CG, Avins AL, Barry MJ, Bruskewitz RC, Donnell RF, Foster HE Jr, Gonzalez CM, Kaplan SA, Penson DF, et al: Update on AUA guideline on the management of benign prostatic hyperplasia. J Urol 185: 1793-1803, 2011.

This work is licensed under a Creative Commons Attribution-NonCommercial-NoDerivatives 4.0 International (CC BY-NC-ND 4.0) License. 